\title{
Epstein-Barr virus and telomerase: from cell immortalization to therapy
}

\author{
Riccardo Dolcetti ${ }^{1 *}$, Silvia Giunco ${ }^{2}$, Jessica Dal Col ${ }^{1}$, Andrea Celeghin ${ }^{2}$, Katy Mastorci ${ }^{1}$ and Anita De Rossi ${ }^{2}$
}

\begin{abstract}
Overcoming cellular senescence is strictly required for virus-driven tumors, including those associated with EpsteinBarr virus (EBV). This critical step is successfully accomplished by EBV through TERT expression and telomerase activation in infected cells. We herein review the complex interplay between EBV and TERT/telomerase in EBVdriven tumorigenesis. Evidence accumulated so far clearly indicates that elucidation of this issue may offer promising opportunities for the design of innovative treatment modalities for EBV-associated malignancies. Indeed, several therapeutic strategies for telomerase inhibition have been developed and are being investigated in clinical trials. In this respect, our recent finding that TERT inhibition sensitizes EBV+ lymphoma cells to antivirals through activation of EBV Iytic replication is particularly promising and provides a rationale for the activation of clinical studies aimed at assessing the effects of combination therapies with TERT inhibitors and antivirals for the treatment of EBV-associated malignancies.
\end{abstract}

Keywords: Epstein-Barr virus, Telomerase, Cancer, Lymphoma, Lytic replication, Antivirals

\section{Introduction}

More than $90 \%$ of the world's population is infected by Epstein-Barr virus (EBV), a ubiquitous gammaherpesvirus that may silently persist in memory B lymphocytes. Primary infection is usually asymptomatic and when it is delayed until adolescence or adulthood a benign lymphoproliferative disease, known as infectious mononucleosis, may occur. B lymphocytes are the main target of EBV infection in vivo, although epithelial cells, and T or Natural Killer (NK) cells may also carry the virus. Infection of B lymphocytes is usually non-productive or latent, whereas intermittent reactivation and virus replication at epithelial surfaces allow the spreading of EBV to new hosts. Despite its widespread diffusion and apparent harmlessness, EBV is causally linked to the development of both lymphoid and epithelial malignancies, including Burkitt lymphoma (BL), Hodgkin lymphoma, post-transplant lymphoproliferations, AIDS-associated lymphomas, nasopharyngeal and gastric carcinoma [1]. EBV infection of primary B lymphocytes results in permanent growth of these cells, an effect promoted by the full spectrum of EBV-encoded latency proteins, including

\footnotetext{
* Correspondence: rdolcetti@cro.it

${ }^{1}$ Cancer Bio-Immunotherapy Unit, CRO Aviano, National Cancer Institute, Aviano, PN, Italy

Full list of author information is available at the end of the article
}

six EBV nuclear antigens (EBNAs) and three latent membrane proteins (LMP-1, LMP-2A, LMP-2B), the so called latency III program, which can be found in EBVtransformed lymphoblastoid cell lines (LCLs), in posttransplant lymphoproliferations and in AIDS-associated immunoblastic lymphomas [1,2]. A second latency program (type II) can be detected in tumor cells of Hodgkin lymphoma and in nasopharyngeal carcinoma, which express only EBNA-1 and the LMPs, whereas the most restricted form of EBV latency is characteristic of $\mathrm{BL}$ lymphoma in which only EBNA-1 is expressed. In all forms of latency, EBV expresses the EBERs, small nonpolyadenylated, non-coding double-strand RNAs, which may also contribute to EBV-driven B-cell immortalization [3]. EBV may activate the lytic replication program upon terminal differentiation of EBV-infected memory B lymphocytes into antibody-secreting plasma cells. Although well equipped to promote the growth of B lymphocytes, EBV may drive the proliferation of these cells only transiently in immunocompetent hosts. This is due to the existence of a complex, strictly regulated immunological control involving various humoral and cellular effectors of immunity. Through the long time evolutionary adaptation in humans, the virus has evolved several potent mechanisms by which the type III cells that express the growth program evade the immune response. This may also 
explain why only a limited proportion of EBV-seropositive individuals develop EBV-associated lymphomas, even in the setting of immune deficiency.

Studies carried out with recombinant EBV strains lacking individual latent genes allowed the identification of the genes that are essential for B-cell immortalization in vitro. Available evidence indicates that EBNA-2 and LMP-1 are absolutely required for EBV-mediated B-cell transformation, whereas a crucial role is played by EBNA1, EBNA-3, -5, and -6 [1]. Full immortalization is achieved through the concerted action of several EBV proteins that derange cellular pathways controlling growth and/or survival. These viral proteins usually act cooperatively and may induce different biologic effects in different cellular backgrounds. LMP-1 is considered the major EBV oncoprotein, acting as an oncogene in rodent fibroblast cells [4,5]. LMP-1 functions as a constitutively active tumor necrosis factor receptor, mimicking an activated CD40 receptor, although structurally different [6,7]. LMP-1 has pleiotropic functions being able to promote B-cell activation, homotypic and heterotypic cell adhesion and the expression of cell surface (i.e. CD23, CD39, CD40 and CD44) and adhesion (LFA1, ICAM1 and LFA3) molecules. LMP-1 is also responsible for the up-regulation of antiapoptotic proteins, and may suppress cellular senescence. Particularly relevant from an oncogenic point of view is the ability of LMP-1 to activate multiple cellular signaling pathways, including mitogen-activated protein kinase (MAPK), c-Jun N-terminal kinase (JNK), phosphatidylinositol 3kinase (PI3K)/Akt, and NF-KB [8].

LCLs obtained by EBV infection in vitro of B lymphocytes, is considered a useful model to investigate the relationships between virus and host in the EBV-driven lymphomagenesis. Indeed, these immortalized cells are similar to those regularly produced in vivo but negatively controlled by EBV-specific CD8+ cytotoxic T cells [9]. Studies using this model assess that EBV-driven malignancies and LCLs selectively express latent viral proteins and maintain their ability to grow indefinitely through inappropriate activation of TERT. Expression of latent EBV proteins is not sufficient to fully immortalize EBV-infected B cells. Only EBV-carrying B cells with sustained telomerase activity are truly immortalized, whereas telomerasenegative cells, although exhibiting a prolonged life-span, eventually undergo cellular senescence and terminate their life span through the shortening of their telomeres [10,11]. The finding that the majority of EBV-driven tumors in vivo are telomerase-positive confirms the relevance of telomerase expression in the process of tumorigenesis.

\section{Pathogenic role of telomerase in cell immortalization and transformation}

Genetic instability is a hallmark of cancer and tumor cells should circumvent replicative senescence and acquire the ability to sustain unlimited proliferation. Telomere/telomerase interplay is an important mechanism involved in the genomic stability and cellular replicative potential and its dysfunction has emerged as playing a key role in carcinogenesis [12]. Telomeres are specialized DNA structures located at the end of chromosomes and are essential in stabilizing chromosomes by protecting them from endto-end fusion and DNA degradation. Telomeres are composed of (TTAGGG) $n$ tandem repeats associated with the telomere-binding proteins TRF1, TRF2, RAP1, TIN2, TPP1 and POT1, which constitute the shelterin complex [13]. Telomeres are progressively shortened during each cell division by replication-dependent loss of sequences at DNA termini due to the failure of DNA polymerase to completely replicate the $3^{\prime}$ end of chromosomes [14]. When telomeres become critically short (the Hayflick limit), cells undergo replicative senescence and apoptosis; further erosion of telomeres may impair their function in protecting chromosome ends, resulting in genetic instability. Nonetheless, cell division-associated telomere shortening prevents unlimited cell proliferation and, thus, tumour development/progression. To escape this proliferation barrier, cells must stabilize their telomeres. Most tumors maintain their ability to grow indefinitely through inappropriate expression of telomerase, a ribonucleoprotein complex containing an internal RNA template (TR), used as a template for elongation of telomeres, and the protein with telomere-specific reverse transcriptase activity (telomerase reverse transcriptase [TERT]) [15]. While TR has broad tissue distribution and is constitutively present in normal and tumour cells, TERT is the rate-limiting component of the telomerase complex, and its expression generally well correlates with telomerase activity. Overexpression TR along with TERT may increase telomerase activity, while specific TR variants may reduce its association with TERT, thus diminishing the telomerase activity in telomere lengthening [16]. Expression of TERT is generally restricted to stem cells, and is usually repressed in normal somatic cells. It may be expressed at low levels in normal hematopoietic cells according to their state of differentiation/activation. In contrast, TERT is expressed in the vast majority of immortalized and fully transformed cells. TERT is essential for unlimited cell growth, and thus plays a critical role in tumor formation and progression (reviewed in [17]).

Regulation of telomerase operates at several biological levels: transcription, mRNA splicing, sub-cellular localization of each component, and assembly of TR and TERT in an active ribonucleoprotein complex. Transcription of TERT gene is likely the key determinant in the regulation of telomerase activity; TERT transcriptional activity is specifically up-regulated in cancer cells, but it is silent in most normal cells. More than 20 transcription factor-binding sites acting as activators or repressors have been identified 
within the TERT promoter. Cooperation of MYC and SP1 is required for full activation of the TERT promoter, while TP53, through its interaction with SP1, down-regulates TERT. TERT is also directly activated by nuclear factor (NF)-kB, hypoxia-inducible factor-1, and the ETS/MYC complex. The histone methyltransferase SMYD3 also directly contributes to inducible and constitutive TERT expression in normal and malignant human cells. TERT expression is suppressed by the oncosuppressor genes WT127 and MEN1, and through the MAD/MYC and the TGF- $\beta$ /SMAD pathways. Cell cycle inhibitors p16INK4a and p27KIP1 have also been shown to down-regulate TERT expression in cancer cells (reviewed in [17]). Regulation of TERT transcription may also involve DNA methylation, as the TERT promoter contains a cluster of CpG sites. At post-transcriptional level, modulation of telomerase may occur by alternative splicings; at least 10 different variants of TERT mRNA have been described, and some of these splicing products have been proposed to exert a dominant negative function by competitive interaction with components of the telomerase complex [18]. Telomerase activity is also controlled through posttranslational modifications of the TERT protein. Phosphorylation of the protein at critical sites by the PI3K/ AKT kinase pathway seems to be crucial for telomerase activity. Telomere-binding proteins, Telomeric Repeat binding Factor (TRF)1, TRF2, Repressor/Activator Protein1 (RAP1), TRF1-interacting Nuclear protein 2 (TIN2), TTP1 (also known as TINT1, PTOP, PIP1), and Protection Of Telomers 1 (POT1), which constitute the shelterin complex, play a role in the activity of telomerase; TPP1 heteromerizes with POT1; the POT1-TPP1 complex is capable of recruiting and stimulating telomerase activity, thereby regulating telomere length through TPP1telomerase interaction [19]. Notably, recent studies have suggested that, besides maintenance of telomere length, TERT is involved in several other cell functions. Expression of TERT increases replicative kinetics [20,21], promotes cell growth in adverse conditions and may also act as an anti-apoptotic agent [22-24]. High levels of telomerase confer resistance to several antineoplastic drugs $[25,26]$.

\section{Interplay between EBV infection and telomerase activation in EBV-driven tumors}

Although it is well recognized that the establishment of latent EBV infection and TERT activation are both required for EBV-driven cell transformation, temporal and possible causal relationships between these two events remain to be clarified. Early passages EBV-infected B lymphocytes greatly differed in their timing of TERT expression and telomerase activation; EBV-driven B-cell activation may fail in the induction of telomerase activity and telomerase-negative EBV-infected B cells may have a prolonged life-span compared to normal B lymphocytes
$[10,20]$. Although EBV-infected B cells exhibit higher proliferative activity than resting primary $B$ lymphocytes, very few EBV-carrying $B$ cells will eventually progress to immortalization, most of them reaching proliferative crisis and ending their lifespan even after 150 population doubling levels depending on genetic factors, including telomere length. Only LCLs developing a strong telomerase activity associated with aneuploidy overcome cellular crisis and become stably immortalized. Therefore additional changes at the cellular level are required to cooperate with the latent EBV proteins during immortalization of EBV-infected B cells [10]. It has been recently suggested that telomere length may be maintained in EBV-infected B lymphocytes by an alternative lengthening of telomeres (ALT) [27]. However, lack of TERT activation precluded their long-term establishment in culture and their immortalization [20].

In primary $B$ lymphocytes, activation of TERT occurs concomitantly with the induction of latent EBV proteins and down-regulation of EBV lytic gene expression [20]. We have demonstrated that LMP-1 activates TERT at the transcriptional level via NF-kB and MAPK/ERK1/2 pathways [28]. LMP-1 induces telomerase activity also in nasopharyngeal carcinoma cells, an epithelial tumor closely associated with EBV infection. In these cells, LMP-1 up-regulates telomerase expression and phoshorylation through the AKT pathway [29]. In epithelial cells, TERT expression may be also MYC-dependent since mutagenesis of MYC-responsive E-box elements in the TERT promoter inhibited TERT transactivation induced by LMP-1 [30]. In B cells, however, MYC is not involved in mediating the hTERT expression and telomerase activation induced by LMP-1, since MYC silencing does not inhibit LMP-1-induced telomerase activation, and mutagenesis in the NF- $\mathrm{kB}$ binding sites, but not in the MYC binding sites, inhibits LMP-1-induced activation of the TERT promoter [28]. This is of particular interest considering that, while in EBV-negative BL the translocated and over-expressed MYC plays a key role in TERT activation, in EBV-positive diffuse large B-cell Lymphomas and immunoblastic lymphomas where $M Y C$ is in germline configuration, TERT is likely to be activated by LMP-1, as it occurs in LCLs [28].

Notably, in addition to maintaining telomere length and allowing EBV-infected cells to overcome senescence and apoptosis, TERT may promote EBV-driven lymphomagenesis through extra-telomeric functions. One interesting result achieved in vitro using the LCL model is that TERT expression plays a relevant role in inhibiting the virus lytic cycle, thereby favouring the induction and maintenance of EBV latency in primary B lymphocytes, a prerequisite for EBV-driven transformation. Indeed, high level of endogenous TERT expression or ectopic TERT expression on telomerase-negative EBV-infected 
cells prevents the induction of viral lytic cycle. By contrast, TERT silencing by specific siRNA or short-hairpin (sh)RNA induced the expression of BZLF1, EA-D, and gp350 EBV lytic proteins and triggered a complete lytic cycle. This occurs in both EBV-immortalized LCL and fully transformed EBV-positive BL cell lines thus supporting the notion that TERT is a critical regulator of the balance between viral latent and lytic cycles [20,31]. Moreover, TERT inhibition induced an accumulation of cells in the S phase, an effect likely due to the dephosphorylation of 4EBP1, an AKT1-dependent substrate, which results in a decreased availability of proteins needed for cell cycle progression. Besides inducing cell death through activation of complete EBV lytic replication, TERT inhibition triggered AKT1/FOXO3/NOXAdependent apoptosis in EBV-positive and EBV-negative BL cell lines [31].

The fine mechanism(s) by which TERT prevents the expression of lytic proteins is still an interesting open question. It has been demonstrated that the treatment of primary EBV-positive BL with zidovudine (AZT), a thymidine analog, induced EBV lytic cycle and cell death through the NF- $\mathrm{kB}$ pathway [32,33]. Given that AZT may inhibit telomerase activity [34], this finding further supports the strong relationship between TERT level and EBV latent/lytic status and it may suggest that this occurs via NF- $\mathrm{kB}$ pathway. To shed light on the possible mechanisms underlying the activation of EBV lytic replication induced by TERT inhibition, a study investigated the involvement of BATF, a transcription factor, which negatively regulates AP-1 activity [35,36]. BATF has been shown to inhibit the expression of BZLF1, thus reducing EBV lytic replication in latently infected B cells [37]. It has been shown that ectopic expression of TERT in $B$ cells significantly increased BATF expression, whereas
TERT silencing by shRNA decreased BATF mRNA levels and protein expression and induced the expression of lytic protein [31]. These results suggest that TERT silencing promotes the activation of EBV replication by reducing inhibition of BATF-driven BZLF1 transcription (Figure 1). As viral lytic replication is associated with the death of infected cells, TERT inhibition may be promising strategy to treat EBV-driven malignancies.

\section{Telomerase as a promising therapeutic target for human tumors}

The high expression of TERT in tumor cells and the requirement of a sustained telomerase activity for their unlimited proliferation capability make telomerase a particularly attractive target for cancer therapy. Moreover, the fact that rapidly proliferating cancer cells have shorter telomeres compared to normal somatic cells and stem cells further corroborates the enhanced specificity for tumor cells of the cytotoxic effects exerted by drugs targeting telomerase [38]. Several strategies targeting telomerase are being explored at the pre-clinical level, and two major approaches are currently under clinical investigation. Telomerase can be directly targeted by drugs able to inhibit TERT activity or its RNA template. Alternatively, drugs such as G-quadruplex stabilizers, tankyrases or HSP90 inhibitors may act indirectly on telomerase by preventing its access to telomeres or inhibiting binding of telomerase-associated proteins leading to telomere uncapping and cell apoptosis [39]. GRN163L (Imetelstat) is a highly promising drug able to inhibit telomerase activity by acting as a direct telomerase RNA template antagonist rather than behaving as a normal antisense oligonucleotide [40]. This compound has been successfully tested in several phase I studies involving patients with non-small cell lung cancer, locally recurrent
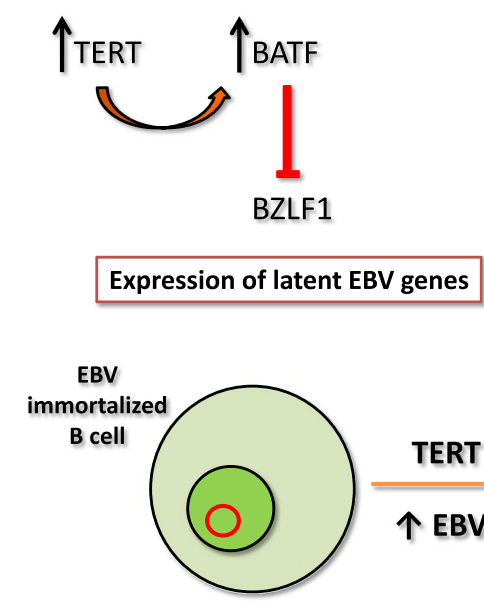

TERT inhibition $\uparrow$ EBV lytic cycle

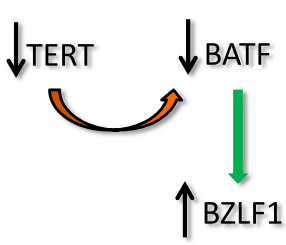

Expression of lytic EBV genes

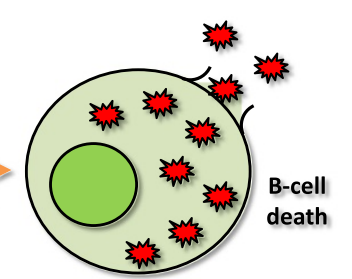

Figure 1 TERT inhibition induces EBV lytic cycle. In EBV-infected B cells, TERT up-regulates the expression of BATF, a negative regulator of BZLF1, thus preserving EBV latency. Following TERT inhibition, BATF level significantly decreases; the expression of BZLF1 leads to the induction of lytic proteins and a complete EBV lytic cycle. 
or metastatic breast cancer, multiple myeloma or essential thrombocythemia and its efficacy is now being investigated in phase II clinical trials [39]. Among small molecule inhibitors targeting hTERT, the non-nucleosidic compound BIBR1532 is particularly interesting due to its ability to inhibit telomerase by non-competitively binding to the active site of TERT [41]. Preclinical studies carried out with cell lines of different tumor histotypes demonstrated that BIBR1532 can inhibit telomerase activity and induce cell growth arrest without causing acute cytotoxicity. Evidence has been also provided indicating that BIBR1532 can sensitize tumor cells to chemotherapy $[42,43]$. T-oligo is a newly developed drug composed of a single stranded 11-bp oligonucleotide with sequence homology to telomeres able to potently induce DNA damage responses including apoptosis, differentiation and senescence $[44,45]$. Notably, in normal cells, T-oligo induces only transient antiproliferative effects due to the presence of functional cell cycle check points [45]. Given that telomere length depends on the balance between progressive loss during cell proliferation and extension induced by telomerase activation, a deeper knowledge of telomere/ telomerase interplay is critical to choose the most appropriate timing for administering telomerase-targeted drugs. This is a highly relevant issue to design the most effective schedules of treatment including these innovative drugs.

A second main therapeutic approach involves immunotherapy strategies targeting telomerase [39]. Considering that most cancers express telomerase, peptides derived by this protein can be considered as universal tumor-associated antigens. Indeed, evidence accumulated so far clearly indicates that telomerase-derived peptides may elicit specific CD8+ and CD4+ T cell responses of potential clinical relevance $[46,47]$. Several strategies are being investigated to enhance the immunogenicity of TERT-based vaccines, including the use of adjuvants like GM-CSF or TLR-7 (GV1001 vaccine) [48] or the generation of cryptic peptide vaccines, such as Vx-001 [49]. In these latter vaccines, one amino acid residue of a TERT peptide is replaced for another resulting in a higher affinity to the presenting HLA molecule and a more efficient stimulation of TERT-specific T cells [49]. In addition to these peptide-based vaccines, immunotherapy approaches targeting TERT also include dendritic cell-based vaccines, which do not suffer from the limitations imposed by HLA restriction. GRNVAC1 is a vaccine composed of immature dendritic cells transfected ex vivo with a chimeric mRNA encoding the entire TERT sequence and a portion of the lysosomalassociated membrane protein, which redirects the TERT protein to the lysosome degradation pathway favoring its degradation into immunogenic peptides [50]. Several TERT-targeting vaccines have successfully completed phase I/II studies and are now being investigated in phase III clinical trials.

\section{Strategies exploiting telomerase/TERT inhibition for the treatment of EBV-driven tumors}

The observation that EBV-driven tumors generally express telomerase at high levels provides the rationale supporting the use of the aforementioned pharmacological and immunological approaches targeting telomerase/TERT also for the treatment of these malignancies. Nevertheless, exploitation of telomerase inhibition in this setting may offer additional advantages of potential clinical relevance. In fact, our observation that inhibition of TERT by shRNA triggers virus replication in both EBV-immortalized and fully transformed B cells [31] provides the highly promising opportunity to combine TERT inhibition with antiviral drugs to improve the rate of clinical responses. Indeed, there is an increasing interest in developing strategies able to reactivate EBV lytic gene expression in latently infected tumor cells for the treatment of overt EBV-associated lymphomas. In fact, lytic infection may promote the death of EBV-positive lymphoma cells in vivo, an effect that may be therapeutically relevant since it also favours immune recognition of viral antigens that further enhances the killing of tumor cells. Therefore, drugs targeting cells undergoing viral replication may be used not only to prevent or contain the spreading of EBV infection, but also mainly for their cytotoxic activity on the infected cells and the adjacent cells [51]. Several chemotherapeutic drugs are known to trigger EBV replication, and combination of antivirals with lytic cycle inducers is emerging as a promising strategy for the treatment of EBV-driven lymphomas [52]. We have recently demonstrated that ganciclovir markedly enhances the the anti-proliferative and pro-apoptotic effects induced by TERT inhibition in both EBV-positive BL cells and LCLs [31]. This is probably related to induction of EBV replication and may be the result of the activation of the pro-drug by EBV lytic products such as the viral thymidine kinase or viral protein kinase. In this respect, drugs able to inhibit TERT may be regarded as sensitizers for the activity of antivirals. On these grounds, the combination of antiviral drugs with strategies able to inhibit TERT expression/activity may result in therapeutically relevant effects in patients with EBV-related malignancies. This possibility appears particularly promising in light of the recent development of potent and specific telomerase inhibitors.

In EBV-associated tumors, telomerase can be also targeted indirectly by strategies that inhibit the expression of LMP-1, the major viral oncoprotein endowed with pleiotropic effects also including the ability to upregulate TERT at the transcriptional level $[28,29]$. In this respect, it has been demonstrated that inhibition of 
LMP-1 expression by a DNAzyme (Dz1) able to cleave LMP-1 mRNA down-regulates the expression of the catalytic subunit of telomerase (TERT), both at the protein and mRNA levels, and consequently inhibits telomerase activity in LMP1-positive nasopharyngeal carcinoma cells [29]. Similar strategies may be adopted with therapeutic purposes also for EBV-driven lymphomas.

\section{Conclusions}

Available evidence indicates that TERT expression and telomerase activation play a critical role in EBV-driven tumorigenesis. Several therapeutic strategies for telomerase inhibition have been developed and are being investigated in clinical trials, although data concerning the response rates obtained in patients with EBV-associated lymphomas are limited. Most therapeutics have shown to be more effective when used in combination with standard therapies. In this respect, our finding that TERT inhibition sensitizes EBV+lymphoma cells to antivirals through activation of EBV lytic replication is particularly promising and provides a rationale for conducting further research to assess the effects of combination therapies with TERT inhibitors and antivirals for the treatment of EBV-associated malignancies.

\section{Abbreviations}

AZT: Zidovudine; ATL: Alternative lengthening of telomerases; BL: Burkitt lymphoma; EBV: EBV nuclear antigens; EBNAs: Epstein-Barr virus;

LCL: Lymphoblastoid cell lines; MAPK: Mitogen-activated protein kinase; NK: Natural killer; NF: Nuclear factor; JNK: c-Jun N-terminal kinase; PI3K: Phosphatidylinositol 3-kinase; sh: Short-hairpin; TERT: Telomerase reverse transcriptase.

\section{Competing interests}

The authors declare that they have no competing interests.

\section{Authors' contributions}

All authors performed the literature research, composed the article and approved the final version to be submitted. All authors read and approved the final manuscript.

\section{Acknowledgements}

The study was supported in part by grants from the Associazione Italiana per la Ricerca sul Cancro (Contract 14287 to R.D. and Contract 14258 to A.D.R.).

\section{Author details}

${ }^{1}$ Cancer Bio-Immunotherapy Unit, CRO Aviano, National Cancer Institute, Aviano, PN, Italy. ${ }^{2}$ Viral oncology Unit, Section of Oncology and Immunology, Department of Surgery, Oncology and Gastroenterology, University of Padova, IOV-IRCCS, Padova, Italy.

Received: 18 December 2013 Accepted: 24 February 2014 Published: 26 February 2014

\section{References}

1. Dolcetti R, Dal Col J, Martorelli D, Carbone A, Klein E: Interplay among viral antigens, cellular pathways and tumor microenvironment in the pathogenesis of EBV-driven lymphomas. Semin Cancer Biol 2013, 23(6):441-456

2. Gloghini A, Dolcetti R, Carbone A: Lymphomas occurring specifically in HIV-infected patients: from pathogenesis to pathology. Semin Cancer Biol 2013, 23(6):457-467.
3. Yajima M, Kanda T, Takada K: Critical role of Epstein-Barr Virus (EBV)encoded RNA in efficient EBV-induced B-lymphocyte growth transformation. J Virol 2005, 79(7):4298-4307.

4. Wang D, Liebowitz D, Kieff E: An EBV membrane protein expressed in immortalized lymphocytes transforms established rodent cells. Cell 1985, 43(3 Pt 2):831-840

5. Kaye KM, Izumi KM, Kieff E: Epstein-Barr virus latent membrane protein 1 is essential for B-lymphocyte growth transformation. Proc Natl Acad Sci USA 1993, 90(19):9150-9154.

6. Mosialos G, Birkenbach M, Yalamanchili R, VanArsdale T, Ware C, Kieff E: The Epstein-Barr virus transforming protein LMP1 engages signaling proteins for the tumor necrosis factor receptor family. Cell 1995, 80(3):389-399.

7. Eliopoulos AG, Dawson CW, Mosialos G, Floettmann JE, Rowe M, Armitage RJ, Dawson J, Zapata JM, Kerr DJ, Wakelam MJ, Reed JC, Kieff E, Young LS: CD40-induced growth inhibition in epithelial cells is mimicked by Epstein-Barr Virus-encoded LMP1: involvement of TRAF3 as a common mediator. Oncogene 1996, 13(10):2243-2254.

8. Young LS, Rickinson AB: Epstein-Barr virus: 40 years on. Nat Rev Cancer 2004, 4(10):757-768.

9. Gudgeon NH, Taylor GS, Long HM, Haigh TA, Rickinson AB: Regression of Epstein-Barr virus-induced B-cell transformation in vitro involves virusspecific CD8+ T cells as the principal effectors and a novel CD4+ T-cell reactivity. J Virol 2005, 79(9):5477-5488.

10. Sugimoto M, Tahara H, Ide T, Furuichi Y: Steps involved in immortalization and tumorigenesis in human B-lymphoblastoid cell lines transformed by Epstein-Barr virus. Cancer Res 2004, 64(10):3361-3364.

11. Jeon JP, Nam HY, Shim SM, Han BG: Sustained viral activity of epstein-Barr virus contributes to cellular immortalization of lymphoblastoid cell lines. Mol Cells 2009, 27(2):143-148.

12. Blackburn EH, Greider CW, Szostak JW: Telomeres and telomerase: the path from maize, Tetrahymena and yeast to human cancer and aging. Nat Med 2006, 12(10):1133-1138.

13. Palm W, de Lange T: How shelterin protects mammalian telomeres. Annu Rev Genet 2008, 42:301-334.

14. Harley $C B$, Futcher $A B$, Greider $C W$ : Telomeres shorten during ageing of human fibroblasts. Nature 1990, 345(6274):458-460.

15. Kim NW, Piatyszek MA, Prowse KR, Harley CB, West MD, Ho PL, Coviello GM, Wright WE, Weinrich SL, Shay JW: Specific association of human telomerase activity with immortal cells and cancer. Science 1994, 266(5193):2011-2015.

16. Robart AR, Collins K: Investigation of human telomerase holoenzyme assembly, activity, and processivity using disease-linked subunit variants. J Biol Chem 2010, 285(7):4375-4386.

17. Dolcetti R, De Rossi A: Telomere/telomerase interplay in virus-driven and virus-independent lymphomagenesis: pathogenic and clinical implications. Med Res Rev 2012, 32(2):233-253.

18. Saeboe-Larssen S, Fossberg E, Gaudernack G: Characterization of novel alternative splicing sites in human telomerase reverse transcriptase (hTERT): analysis of expression and mutual correlation in mRNA isoforms from normal and tumour tissues. BMC Mol Biol 2006, 7:26.

19. Nandakumar J, Bell CF, Weidenfeld I, Zaug AJ, Leinwand LA, Cech TR: The TEL patch of telomere protein TPP1 mediates telomerase recruitment and processivity. Nature 2012, 492(7428):285-289.

20. Terrin L, Dolcetti R, Corradini I, Indraccolo S, Dal Col J, Bertorelle R, Bonaldi L, Esposito G, De Rossi A: hTERT inhibits the Epstein-Barr virus lytic cycle and promotes the proliferation of primary B lymphocytes: implications for EBV-driven lymphomagenesis. Int J Cancer 2007, 121(3):576-587.

21. Rampazzo E, Bonaldi L, Trentin L, Visco C, Keppel S, Giunco S, Frezzato F, Facco M, Novella E, Giaretta I, Del Bianco P, Semenzato G, De Rossi A: Telomere length and telomerase levels delineate subgroups of B-cell chronic lymphocytic leukemia with different biological characteristics and clinical outcomes. Haematologica 2012, 97(1):56-63.

22. Del Bufalo D, Rizzo A, Trisciuoglio D, Cardinali G, Torrisi MR, ZangemeisterWittke U, Zupi G, Biroccio A: Involvement of hTERT in apoptosis induced by interference with Bcl-2 expression and function. Cell Death Differ 2005, 12(11):1429-1438.

23. Rahman R, Latonen L, Wiman KG: hTERT antagonizes p53-induced apoptosis independently of telomerase activity. Oncogene 2005, 24(8):1320-1327

24. Jin X, Beck S, Sohn YW, Kim JK, Kim SH, Yin J, Pian X, Kim SC, Choi YJ, Kim H: Human telomerase catalytic subunit (hTERT) suppresses p53-mediated 
anti-apoptotic response via induction of basic fibroblast growth factor. Exp Mol Med 2010, 42(8):574-582.

25. Tao SF, Zhang CS, Guo XL, Xu Y, Zhang SS, Song JR, Li R, Wu MC, Wei LX: Anti-tumor effect of 5-aza-2'-deoxycytidine by inhibiting telomerase activity in hepatocellular carcinoma cells. World J Gastroenterol 2012, 18(19):2334-2343.

26. Shawi M, Chu TW, Martinez-Marignac V, Yu Y, Gryaznov SM, Johnston JB, Lees-Miller SP, Assouline SE, Autexier C, Aloyz R: Telomerase contributes to fludarabine resistance in primary human leukemic lymphocytes. PLoS One 2013, 8(7):e70428.

27. Kamranvar SA, Chen X, Masucci MG: Telomere dysfunction and activation of alternative lengthening of telomeres in B-lymphocytes infected by Epstein-Barr virus. Oncogene 2013, 32(49):5522-5530.

28. Terrin L, Dal Col J, Rampazzo E, Zancai P, Pedrotti M, Ammirabile G, Bergamin S, Rizzo S, Dolcetti R, De Rossi A: Latent membrane protein 1 of Epstein-Barr virus activates the hTERT promoter and enhances telomerase activity in B lymphocytes. J Virol 2008, 82(20):10175-10187.

29. Yang L, Xu Z, Liu L, Luo X, Lu J, Sun L, Cao Y: Targeting EBV-LMP1 DNAzyme enhances radiosensitivity of nasopharyngeal carcinoma cells by inhibiting telomerase activity. Cancer Biol Ther 2013, 15:1

30. Yang J, Deng X, Deng L, Gu H, Fan W, Cao Y: Telomerase activation by Epstein-Barr virus latent membrane protein 1 is associated with c-Myc expression in human nasopharyngeal epithelial cells. J Exp Clin Cancer Res 2004, 23(3):495-506.

31. Giunco S, Dolcetti R, Keppel S, Celeghin A, Indraccolo S, Dal Col J, Mastorci K, De Rossi A: hTERT inhibition triggers Epstein-Barr virus lytic cycle and apoptosis in immortalized and transformed B cells: a basis for new therapies. Clin Cancer Res 2013, 19(8):2036-2047.

32. Bayraktar UD, Diaz LA, Ashlock B, Toomey N, Cabral L, Bayraktar S, Pereira D, Dittmer DP, Ramos JC: Zidovudine-based lytic-inducing chemotherapy for Epstein-Barr virus-related lymphomas. Leuk Lymphoma 2013. in press.

33. Kurokawa M, Ghosh SK, Ramos JC, Mian AM, Toomey NL, Cabral L, Whitby D, Barber GN, Dittmer DP, Harrington WJ Jr: Azidothymidine inhibits NF-kappaB and induces Epstein-Barr virus gene expression in Burkitt lymphoma. Blood 2005, 106(1):235-240.

34. Gomez DE, Armando RG, Alonso DF: AZT as a telomerase inhibitor. Front Oncol 2012, 2:113.

35. Dorsey MJ, Tae HJ, Sollenberger KG, Mascarenhas NT, Johansen LM, Taparowsky EJ: B-ATF: a novel human bZIP protein that associates with members of the AP-1 transcription factor family. Oncogene 1995, 11(11):2255-2265.

36. Echlin DR, Tae HJ, Mitin N, Taparowsky EJ: B-ATF functions as a negative regulator of AP-1 mediated transcription and blocks cellular transformation by Ras and Fos. Oncogene 2000, 19(14):1752-1763.

37. Johansen LM, Deppmann CD, Erickson KD, Coffin WFI, Thornton TM Humphrey SE, Martin JM, Taparowsky EJ: EBNA2 and activated Notch induce expression of BATF. J Virol 2003, 77(10):6029-6040.

38. Corey DR: Telomeres and telomerase: from discovery to clinical trials. Chem Biol 2009, 16(12):1219-1223.

39. Ruden $\mathrm{M}$, Puri N: Novel anticancer therapeutics targeting telomerase. Cancer Treat Rev 2013, 39(5):444-456.

40. Roth A, Harley CB, Baerlocher GM: Imetelstat (GRN163L)-telomerase-based cancer therapy. Recent Results Cancer Res 2010, 184:221-234.

41. Pascolo E, Wenz C, Lingner J, Hauel N, Priepke H, Kauffmann I, Garin-Chesa P, Rettig WJ, Damm K, Schnapp A: Mechanism of human telomerase inhibition by BIBR1532, a synthetic, non-nucleosidic drug candidate. J Biol Chem 2002, 277(18):15566-15572.

42. Chen H, Li Y, Tollefsbol TO: Strategies targeting telomerase inhibition. Mol Biotechnol 2009, 41(2):194-199.

43. Parsch D, Brassat U, Brummendorf TH, Fellenberg J: Consequences of telomerase inhibition by BIBR1532 on proliferation and chemosensitivity of chondrosarcoma cell lines. Cancer Invest 2008, 26(6):590-596.

44. Eller MS, Puri N, Hadshiew IM, Venna SS, Gilchrest BA: Induction of apoptosis by telomere 3' overhang-specific DNA. Exp Cell Res 2002, 276(2):185-193.

45. Longe HO, Romesser PB, Rankin AM, Faller DV, Eller MS, Gilchrest BA, Denis GV: Telomere homolog oligonucleotides induce apoptosis in malignant but not in normal lymphoid cells: mechanism and therapeutic potential. Int J Cancer 2009, 124(2):473-482.

46. Shay JW, Keith WN: Targeting telomerase for cancer therapeutics. $\mathrm{Br} J$ Cancer 2008, 98(4):677-683.
47. Beatty GL, Vonderheide RH: Telomerase as a universal tumor antigen for cancer vaccines. Expert Rev Vaccines 2008, 7(7):881-887.

48. Shaw VE, Naisbitt DJ, Costello E, Greenhalf W, Park BK, Neoptolemos JP, Middleton GW: Current status of GV1001 and other telomerase vaccination strategies in the treatment of cancer. Expert Rev Vaccines 2010, 9(9):1007-1016.

49. Bolonaki I, Kotsakis A, Papadimitraki E, Aggouraki D, Konsolakis G, Vagia A, Christophylakis C, Nikoloudi I, Magganas E, Galanis A, Cordopatis P, Kosmatopoulos K, Georgoulias V, Mavroudis D: Vaccination of patients with advanced non-small-cell lung cancer with an optimized cryptic human telomerase reverse transcriptase peptide. J Clin Oncol 2007, 25(19):2727-2734.

50. Su Z, Vieweg J, Weizer AZ, Dahm P, Yancey D, Turaga V, Higgins J, Boczkowski D, Gilboa E, Dannull J: Enhanced induction of telomerasespecific CD4(+) T cells using dendritic cells transfected with RNA encoding a chimeric gene product. Cancer Res 2002, 62(17):5041-5048.

51. Feng WH, Israel B, Raab-Traub N, Busson P, Kenney SC: Chemotherapy induces lytic EBV replication and confers ganciclovir susceptibility to EBV-positive epithelial cell tumors. Cancer Res 2002, 62(6):1920-1926.

52. Wildeman MA, Novalic Z, Verkuijlen SA, Juwana H, Huitema AD, Tan IB, Middeldorp JM, de Boer JP, Greijer AE: Cytolytic virus activation therapy for epstein-barr virus-driven tumors. Clin Cancer Res 2012, 18(18):5061-5070.

doi:10.1186/1750-9378-9-8

Cite this article as: Dolcetti et al:: Epstein-Barr virus and telomerase: from cell immortalization to therapy. Infectious Agents and Cancer 2014 9:8.

\section{Submit your next manuscript to BioMed Central and take full advantage of:}

- Convenient online submission

- Thorough peer review

- No space constraints or color figure charges

- Immediate publication on acceptance

- Inclusion in PubMed, CAS, Scopus and Google Scholar

- Research which is freely available for redistribution 